\title{
Highly indistinguishable single photons from incoherently excited quantum dots
}

\author{
Marcus Reindl $\odot,{ }^{1, *}$ Jonas H. Weber, ${ }^{2}$ Daniel Huber, ${ }^{1}$ Christian Schimpf, ${ }^{1}$ Saimon F. Covre da Silva, ${ }^{1}$ Simone L. Portalupi, ${ }^{2}$ \\ Rinaldo Trotta, ${ }^{3}$ Peter Michler, ${ }^{2}$ and Armando Rastelli ${ }^{1, \dagger}$ \\ ${ }^{1}$ Institute of Semiconductor and Solid State Physics, Johannes Kepler University Linz, Altenbergerstraße 69, A-4040 Linz, Austria \\ ${ }^{2}$ Institut für Halbleiteroptik und Funktionelle Grenzflächen, Center for Integrated Quantum Science and Technology (IQ $\left.{ }^{S T}\right)$ \\ and SCoPE, University of Stuttgart, Allmandring 3, D-70569 Stuttgart, Germany \\ ${ }^{3}$ Dipartimento di Fisica, Sapienza Universita di Roma, Piazzale Aldo Moro 5, I-00185 Rome, Italy
}

(Received 27 February 2019; revised manuscript received 2 August 2019; published 18 October 2019)

\begin{abstract}
Semiconductor quantum dots with dimensions exceeding the free-exciton Bohr radius are appealing because of their high oscillator strengths. While this property has received much attention in the context of cavity quantum electrodynamics, little is known about the degree of indistinguishability of single photons consecutively emitted by such dots and on the proper excitation schemes to achieve high indistinguishability. A prominent example is represented by GaAs quantum dots obtained by local droplet etching, which recently outperformed other systems as triggered sources of entangled photon pairs. On these dots, we compare different single-photon excitation mechanisms, and we find (i) poor indistinguishability for conventional excitation via excited states and (ii) photon indistinguishablilities above $90 \%$ for both strictly resonant and for incoherent phonon-assisted excitation. Among the explored excitation schemes, optical phonon-assisted excitation allows straightforward laser rejection and is thus worth of further investigation and optimization for quantum dots embedded in high-brightness photonic structures.
\end{abstract}

DOI: 10.1103/PhysRevB.100.155420

\section{INTRODUCTION}

Quantum states of light are the unrivaled resource of future quantum communication networks. In the framework of single-photon emission, epitaxial quantum dots (QDs), such as InGaAs QDs [1,2] or InAsP QDs [3], are well established solid-state sources. In such QDs, the exciton Bohr radius is typically larger than the spatial extensions of the semiconductor heterostructure and the QD's optical properties are largely governed by the confinement potential. The opposite situation, the weak confinement regime, is encountered when the Coloumb interaction between charge carriers begins to overwhelm effects related to the quantum confinement. This regime, which is characterized by enhanced light-matter coupling and hence oscillator strengths [4], has attracted much attention in cavity quantum electrodynamics so as to reach the so-called strong-coupling regime [5]. To our knowledge, the highest oscillator strengths so far have been obtained in "natural" GaAs QDs formed in thickness fluctuations of thin GaAs/AlGaAs quantum wells [6]. In terms of single-photon emission properties, however, QDs in the weak-confinement regime have received limited attention so far since these natural QDs provide poor control on the lateral confinement

\footnotetext{
${ }^{*}$ Corresponding author: marcus.reindl@jku.at

${ }^{\dagger}$ Corresponding author: armando.rastelli@jku.at
}

Published by the American Physical Society under the terms of the Creative Commons Attribution 4.0 International license. Further distribution of this work must maintain attribution to the author(s) and the published article's title, journal citation, and DOI. potential and feature a small energy separation between (discrete) confined states and (continuum) delocalized states. To address this issue while preserving large lateral extensions, different growth protocols have been developed over the years [7-9]. Here we focus on GaAs QDs obtained by droplet etching of nanoholes in $\mathrm{AlGaAs}$ followed by GaAs filling [9], which typically have lateral sizes exceeding the free exciton Bohr radius in GaAs [see Fig. 1(a)]. The excitonic lifetime in this kind of dots $(\approx 250 \mathrm{ps})$ is substantially shorter than the minimum lifetime expected for GaAs QDs in the strong confinement limit $(\approx 440 \mathrm{ps})$ [10], providing strong indication that excitons are weakly confined in our QDs. These QDs enabled the triggered emission of polarization entangled photons at near-unity fidelity [11], partly due to their intrinsically high oscillator strength $[12,13]$. Furthermore, under proper excitation conditions, GaAs QDs provided record low values of multiphoton emission probabilities [14], demonstrating that the weak confinement in these dots does not affect the singlephoton purity. Having these excellent properties at hand, all-photonic quantum teleportation [15] and entanglement swapping [16] schemes were already elaborated. Nonetheless, efficient long-distance quantum networks [17] demand highly indistinguishable photons, which are also essential for photonic quantum information processing $[18,19]$.

Most of the former reports on the indistinguishability of photons subsequently emitted by GaAs QDs were based on resonant two-photon excitation of the biexciton state [12,20,21]. From reports on InGaAs QDs, it is known that the highest values of the visibility of the two-photon Hong-Ou-Mandel (HOM), $V_{\mathrm{HOM}}$, are obtained under strictly resonant excitation (resonant fluorescence (RF) [22]) and that near-unity values can be obtained by applying RF to QDs 
(a)

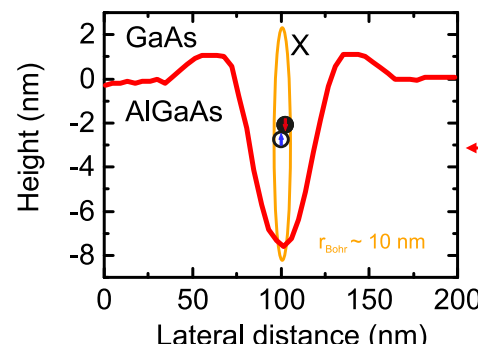

(c)
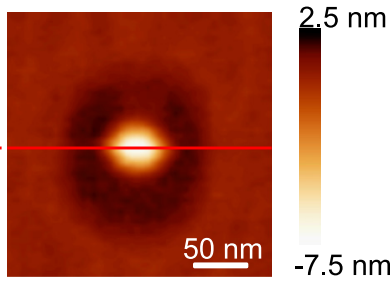

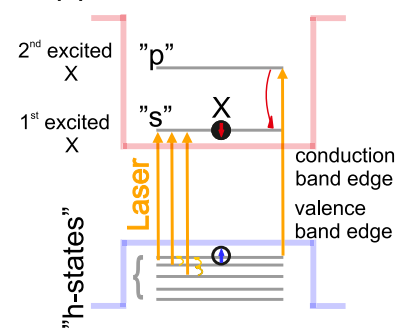

(b)

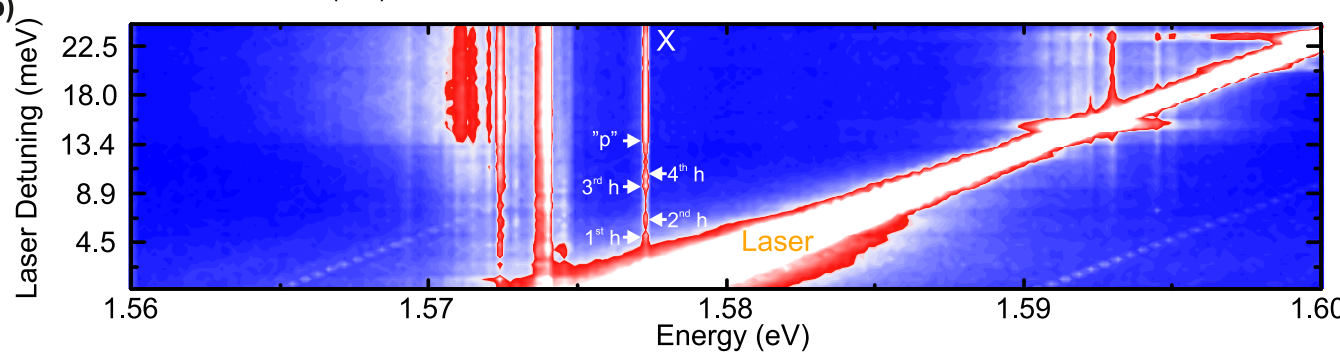

(d)

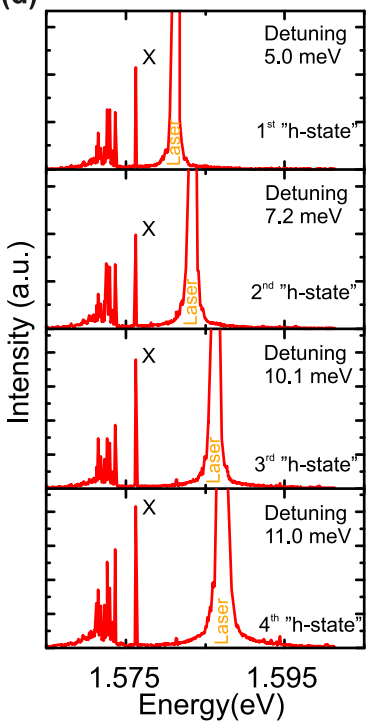

FIG. 1. (a) Atomic force microscopy image of a droplet-etched nanohole in an AlGaAs layer. A line scan across the hole (red) is used to demonstrate the relative wave-function extension of a created exciton with quoted Bohr radius $r_{\text {Bohr }}$. The actual GaAs quantum dot is obtained after filling the hole with GaAs and overgrowth with AlGaAs. (b) Photoluminescence excitation spectroscopy of a typical GaAs quantum dot for positive laser energy detunings with respect to the neutral exciton. High power excitation was used to detect weak resonances. (c) Simplified single-particle sketch of the level structure to visualize the difference between the densely spaced hole states (h states) and the more confined conduction band $s$ and $p$ states. (d) Selected spectra from (b) obtained by tuning the laser wavelength to match the energy of the first, second, third, and fourth excited $\mathrm{h}$ state in the QD valence band to form the neutral X.

embedded into optical microcavities providing simultaneously the Purcell enhancement and spectral filtering [1,2]. Unless special microcavity designs are used [23,24], RF usually requires to sacrifice source efficiency because of the demanding cross-polarized excitation/collection configuration needed for laser rejection.

In this paper, we therefore investigate the limits of photon indistinguishability from droplet-etched GaAs QDs under various excitation conditions to assess the true emitter performance not yet revealed under two-photon pumping and, in particular, focus on practical, thus incoherent driving schemes. Thereby, we do not only compare strictly resonant excitation to the well known excitation via excited states or the LA-phonon excitation $[25,26]$ but also exploit the rarely considered triggered excitation via the LO phonon [27], which yields excellent performance in the given material system.

The reason is the much different level scheme encountered in these weakly confined quantum dots which allows to resonantly dress the LO phonon more independent from other excited states in contrast to standard epitaxial quantum dots. Combined with the fact that the transition rate is intrinsically enhanced in the weak-confinement regime, our results demonstrate the possibility to generate highly indistinguishable photons even for a largely detuned excitation laser-as it is the case for the LO-phonon resonance-and allow insight into different relaxation processes present in droplet-etched GaAs QDs.

\section{METHODS AND RESULTS}

The QD layer is obtained by Al-droplet etching on $\mathrm{Al}_{0.4} \mathrm{Ga}_{0.6}$ As followed by deposition of $2-\mathrm{nm}$ GaAs. With proper optimization, this technique allows the fabrication of highly symmetric QDs [9]. The QD layer is placed at the center of a $\lambda$-cavity consisting of a $\lambda / 2$-thick (123-nm) layer of $\mathrm{Al}_{0.4} \mathrm{Ga}_{0.6} \mathrm{As}$ sandwiched between two $\lambda / 4$-thick $(60-\mathrm{nm})$ $\mathrm{Al}_{0.2} \mathrm{Ga}_{0.8} \mathrm{As}$ layers. The cavity sits on top of a distributed Bragg reflector made of nine pairs of $\lambda / 4$-thick $\mathrm{Al}_{0.95} \mathrm{Ga}_{0.05} \mathrm{As}$ $(70-\mathrm{nm})$ and $\mathrm{Al}_{0.2} \mathrm{Ga}_{0.8} \mathrm{As}$ layers and below two pairs of the same material combination. A 4-nm-thick GaAs protective layer completes the structure. The limited number of pairs used for the DBR mirrors does not yield any measurable Purcell enhancement but an increase in light extraction efficiency. This is important, as it allows us to demonstrate high performance single-photon emission solely related to intrinsic weak confinement effects-boosting the achievable transitions rates - and without the aid of extrinsic light-matter engineering. Finally, a solid immersion lens is placed on top of the grown sample to enhance the external collection efficiency to values up to $12 \%$.

We start our study via pulsed excitation with a laser energetically located exactly at the energy of the neutral exciton (X) of a typical droplet-etched GaAs QD (see supplementary note 1 for technical details [28]). In general, all studies performed here are related to the neutral X. In a next step, we perform photoluminescence excitation spectroscopy (PLE) by detuning the energy of the excitation laser with respect to the $\mathrm{X}$ [see Fig. 1(b)] to identify any relevant resonant population mechanism. Various effects emerge, as discussed in the following.

Excited states of GaAs QDs. First of all, under any positively detuned excitation condition, at least four other lines appear (for sufficient excitation power) on the low-energy side of the $\mathrm{X}$. We attribute the appearance of these lines 
to the excitation of the quantum dot in presence of extra carriers (most probably holes) stemming from residual doping. Because of the slow relaxation (see later in the text), this gives rise not only to ground-state trion emission but also to emission from trions with one extra carrier in an excited state. Further detuning then reveals the presence of resonances (local maxima in the $\mathrm{X}$ intensity) as shown in Figs 1(b) and 1(d). A stable emission pattern is reached at a detuning energy of approximately $13 \mathrm{meV}$, which we identify as the $p$-shell energy. In a single-particle picture, we would attribute the $p$ shell to a configuration featuring one electron in the first excited state in the conduction band. The appearance of several resonances between the electron $s$ and $p$ shells of the QD are instead attributed to densely spaced excited hole states [7], which we denote as "h states." We stress that the single-particle picture described above is an intuitive but poor approximation for the large QDs studied here and that resonances should be simply regarded as excited states of the system. In the weak-confinement regime, we would interpret the densely spaced resonances as excited states of the center-of-mass motion of the exciton in the lateral potential (dominated by the heavier mass of holes) and the $p$ shell as the internal excitation of the exciton (dominated by the lighter mass of the electron).

Decay dynamics under coherent and incoherent resonant excitation. We continue our study with the evaluation of the decay dynamics for all detected $\mathrm{X}$ resonances under excitation with laser pulses having a typical duration of $\approx 3-10 \mathrm{ps}$. First of all, the strictly resonant condition, the coherent excitation of the resonance fluorescence [29], collected using a crosspolarization configuration, reveals a decay time of 209(5) ps under $\pi$-pulse condition [see Fig. 2(a)]. An exemplary spectrum is shown in Fig. 2(b). The visible beating in the decay dynamics is associated to the phase evolution of the fine structure split bright excitons [30,31], a feature which complicates the use of neutral excitons as emitters of polarized single photons under RF excitation. Therefore we investigate more practical incoherent population schemes for positively detuned excitation laser energies. A small detuning of $0.5 \mathrm{meV}$ allows us to excite the $\mathrm{X}$ via the LA-phonon sideband [26] and yields a decay time of 236(8) ps [see Fig. 2(a)], a value compatible with that obtained under RFconsidering that a different QD was used-or under resonant two-photon excitation conditions $[12,13]$. This result is in line with previous works on InGaAs QDs, which indicated that the thermalization of the exciton with the phonon bath occurs on picosecond timescales, i.e., within the duration of our laser pulse [26,32]. An exemplary spectrum obtained under LA-phonon-assisted excitation is shown in Fig. 2(b) and demonstrates a frequently encountered resonant behavior of droplet-etched GaAs QDs at moderate excitation powers: A strongly enhanced $X$ transition accompanied by four suppressed, low-energy lines.

Surprisingly, further detuning towards the resonant condition of the first excited h state on the same QD, just $5 \mathrm{meV}$ detuned from the $X$ [see Fig. 1(d)] reveals a completely different behavior. A much slower rise in the time trace followed by a decay time as long as $1.84(0.04) \mathrm{ns}$ is observed. It is reasonable to assume that the actual radiative lifetime of the $\mathrm{X}$ transition did not change and that the decay is dominated by
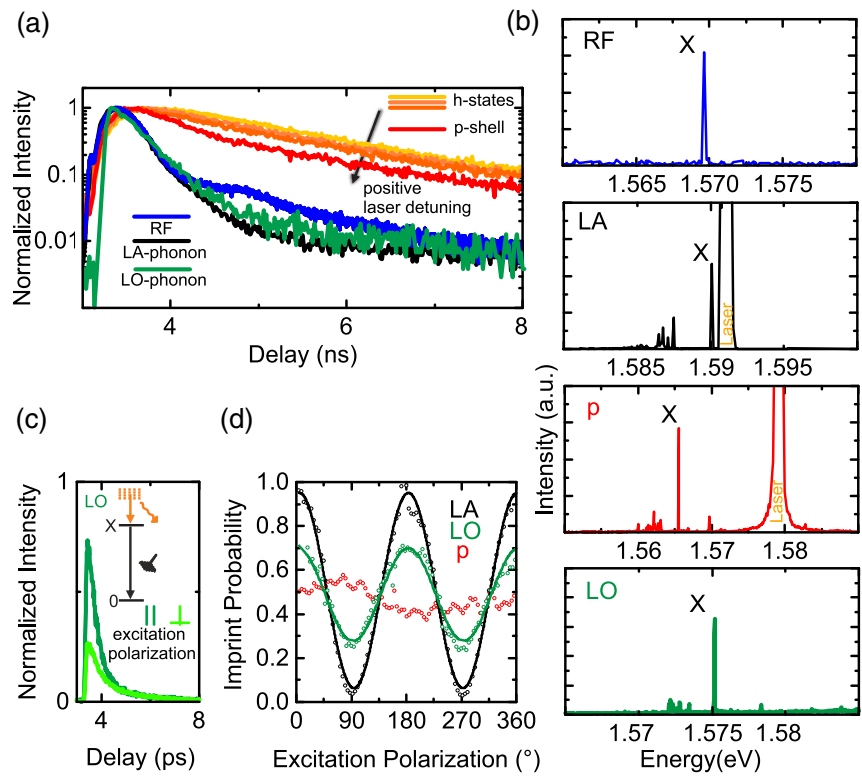

(d)
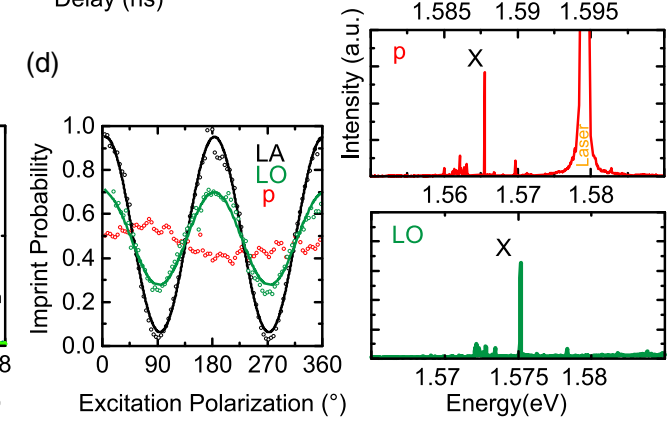

FIG. 2. (a) Time-correlated single-photon counting under various excitation schemes as measured on the $\mathrm{X}$ transition of a GaAs QD for RF excitation (blue), LA-phonon excitation (black), LOphonon excitation (green), $p$-shell excitation (red), and excited $\mathrm{h}$ states (shades of orange). All decay times are extracted via a single (double for LO) exponential fit and under deconvolution of the instruments response function (and phase evolution of the fine structure split X states in RF and LA). (b) Exemplary spectra of coherently and incoherently excited X states: RF excitation (top, blue), LA-phonon excitation (second top, black), $p$-shell excitation (second bottom, red), and the LO-phonon excitation (bottom, green). (c) Decay time measurements on the LO-phonon for a parallel (dark green) and orthogonal (light green) configuration of excitation polarization with respect to the collection polarization fixed to one of the two bright excitonic states. (Inset) Simplified sketch of the created polaron decaying via the emission of one LO phonon and one $\mathrm{X}$ photon. (d) The probability of the polarization transfer from laser photons to the emitted single photons as a function of the excitation polarization angle under LA-phonon excitation (black), LO-phonon excitation (green), and $p$-shell excitation (red). The angle is referred to a fixed polarization in the collection system aligned to one of the two bright excitonic states. The individual data points are fitted with a cosine function.

the slow relaxation of the excited state. The slow relaxation is persistent even under resonant $p$-shell $(\approx 13 \mathrm{meV})$ excitation [see Fig. 2(b)] in these QDs, where we witness decay times of $1.51(0.05) \mathrm{ns}$. This value is still a factor six slower than the time measured under $s$-shell and LA-phonon excitation. We also note that the slow decay persists for optical and electrical above-bandgap excitation $[33,34]$. This slow relaxation might be related to the fact that the energy separation between the hole's excited and ground states $(\approx 5 \mathrm{meV})$ is much smaller than the LO-phonon energy in GaAs (36 meV [35]). In addition to that, the high symmetry of these QDs, reflected by the small, average fine structure splitting (FSS) and negligible light-hole-heavy-hole mixing in the ground-state exciton [9] might lead to rather restrictive orbtial/spin selection rules for the relaxation processes. We note that the relaxation times observed here are longer than those typically observed for 
QDs in the strong confinement regime [36]. In this regime, the excited states have an energy comparable to the LO-phonon energy such that the carrier relaxation times are enhanced due to an anharmonic polaron (carrier coupled to optical phonon) decay as reported in the literature [37-39]. Finally, the observation of long decay times $(\approx 1.5-2 \mathrm{~ns})$ for excitation via excited states makes us confident that the fast decay time $(\approx 200 \mathrm{ps})$ measured under RF and LA-phonon excitation coincides to the radiative decay time and that the contribution of possible nonradiative processes is negligible.

Longitudinal-optical-phonon-assisted excitation. Since slow relaxation introduces a large time jitter in the photon emission, we expect emission of photons with reasonable indistinguishability only under coherent $s$-shell and LA-phonon excitation. However, in these conditions the spectral proximity of the excitation laser is still not ideal. Parallel polarization of excitation and emission, as required for resonant excitation at maximal efficiency, is challenging [23,24], in particular in terms of the single-photon purity, which is a quantity highly vulnerable to scattered laser light. Thus polarization filtering is usually applied at costs of the source efficiency (at least $50 \%$ less). A possible way to circumvent this problem is to detune the excitation laser even more, by approximately $36 \mathrm{meV}$, the already mentioned LO-phonon energy in GaAs. Although the occurrence of resonant absorption at the LO-phonon energy is well known [40], this pumping scheme has been rarely used for single-photon emission [27], possibly because of the strong overlap of this resonance with the $p$ shell of typical QDs in the strong confinement regime. In contrast to that, for the QDs investigated in this work, the LO-phonon resonance is a bit more separated from the excited states of the system, which is the $d$ shell located $39 \mathrm{meV}$ from the neutral $\mathrm{X}$.

A created polaron can now decay via the emission of one LO phonon, leaving the QD populated with one exciton. This allows us to populate the neutral $\mathrm{X}$ similar to the strictly resonant $s$-shell and LA-phonon-assisted excitation schemes, as proven by the measurement of the decay time in Fig. 2(a) with the remarkable benefit of an excitation source being largely detuned with respect to the transition of interest. As a consequence, there is no need of any sophisticated filtering technique. An exemplary spectrum is shown in Fig. 2(b), which resembles the resonant behavior discussed beforehand. We want to emphasize at this point that the ideal resonant situation (dominant $\mathrm{X}$ intensity and vanishing low-energy states) is usually not observed for all excitation conditions on a single QD at the same time. It happens that an efficiently generated $\mathrm{X}$ under LO-phonon excitation shows nonideal resonant behavior under LA-phonon or strict resonant excitation despite observing the same QD, which we attribute to the details of the residual defects/doping configuration in the surroundings of the QD [41]. For this reason, we show in Fig. 2(b) spectra of different QDs, which display the best resonant condition for each of the different excitation schemes.

It is interesting to mention that any detuning from the LO-phonon resonance immediately leads to the reported stagnating recombination times previously shown in Fig. 2(a). From the decay curve obtained under LO-phonon excitation, we extract a lifetime of 251(8) ps accompanied by a slow decay with characteristic time of $2.30(0.11) \mathrm{ns}$. From the fast rise and short decay time, we deduce that the polaron decay occurs on timescales substantially shorter than the lifetime of our emitters and possibly already on timescales comparable to our pulse lengths. We also note that only a negligible amount of photons is found to be related to the slow decay channel if we do not account for photons lost to the low-energy states emission. This slow decay channel clearly resembles the characteristics of the emitter's excited states, meaning that we can not yet perfectly address the LO-phonon resonance (proximity of d-shell) or that the polaron has a finite probability to decay into an exited state exciton plus acoustic phonons. We can easily quantify the loss in efficiency, as the incoherently placed laser allows us to arbitrarily align the excitation polarization to one of the bright excitonic transition components separated by the FSS as demonstrated in Fig. 2(c). Here, we first aligned the analyzing polarizer to one fine structure component of the $\mathrm{X}$ and then compared the temporal decay behavior for parallel and orthogonal excitation configurations. The orthogonal case does reveal a weaker intensity but in the ideal situation this anti-parallel driving scheme should be completely suppressed for the investigated $\mathrm{X}$ transition due to conservation of angular momentum, as the dissipated LO phonon will carry no spin. However, the experimental result indicates the presence of depolarization mechanisms as already suggested by the presence of an additional slow decay channel. For the sample used in the presented study, we can nevertheless "imprint" the laser polarization with a fidelity of $\sim 75 \%$ [see Fig. 2(d)] on the desired transition component, meaning that we are at least $25 \%$ more efficient than under strict resonant conditions, since no polarization rejecting elements are necessary. At least, because it is actually not trivial to drive a certain polarization axis under RF excitation (on the neutral X) to reach the ideal $50 \%$ efficiency condition.

The LA-phonon assisted scheme represents a promising alternative to RF [42] and exhibits a more efficient coupling to the two-level system compared to LO-phonon excitation [see Fig. 2(d)] as a consequence of the small energy difference between acoustic phonon bath and its addressed transition, which does not allow any relaxation via decay paths including excited states. Nonetheless, we want to emphasize that under optimal conditions (parallel polarization configuration) the scattering of laser light is unavoidable and prohibits perfect single-photon properties of the emitter, a circumstance particularly related to the high pump intensity needed in phononassisted excitation schemes.

Before we compare the achievable photon indistinguishabilites of the presented excitation schemes, we want to investigate the LO-phonon excitation in our GaAs QDs in more detail. First of all, we study the power dependence of the decay dynamics represented in Fig. 3(a). As we crank up the laser power, we can identify two distinct decay channels: (i) the desired decay of the created polaron via the fast emission of one LO phonon and the $\mathrm{X}$ photon (characterized by the fast rise and decay times) and (ii) the undesired population of excited states, which then leads to a slow rise and similar recombination times previously seen under excited-state excitation. At excitation powers where the $\mathrm{X}$ intensity is identical to the ones observed under LA-phonon excitation or strict resonant condition, however, only a small fraction of photons $(\leqslant 2 \%)$ can be associated to this long-decay channel. 
(a)

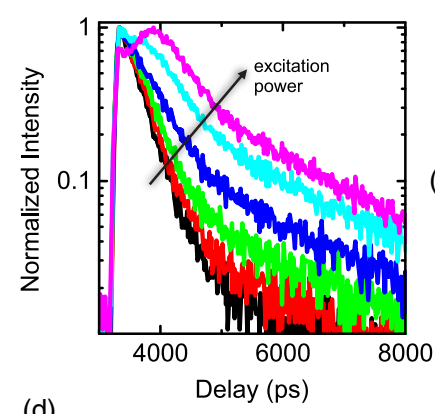

(d)

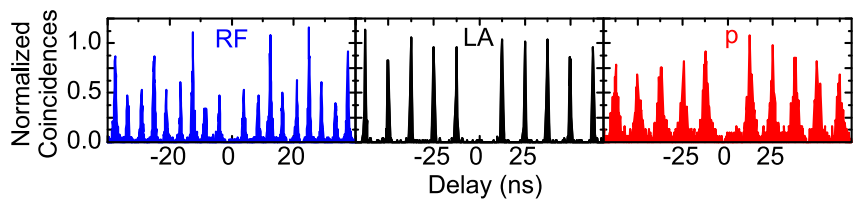

FIG. 3. (a) Power dependent study of the X excitation and decay dynamics under (co-polarized) LO-phonon excitation. (b) Spectral width of the LO-phonon resonance as measured via detuning of the excitation laser. The data points are fitted by a Gaussian function to obtain the quoted FWHM. (c) Second-order correlation function measured on emitted $\mathrm{X}$ photons in a standard HBT setup under the LO-phonon excitation scheme. (d) Additional secondorder correlations measured on emitted $X$ photons under RF excitation $\left[g_{X_{\mathrm{RF}}}^{(2)}(0)=1.9(0.1) \times 10^{-2}\right]$, LA-phonon excitation $\left[g_{X_{\mathrm{LA}}}^{(2)}(0)=\right.$ $\left.2.0(0.1) \times 10^{-2}\right]$, and $p$-shell excitation $\left[g_{X_{p}}^{(2)}(0)=7.3(0.6) \times 10^{-2}\right]$. The histogram counts beyond the laser pulse frequency (under RF) are related to an introduced double excitation time gap of $\Delta t=4 \mathrm{~ns}$.

Next, we tune the energy of the laser in small steps across the LO-phonon resonance to assess its spectral width. Using a Gaussian fit [see Fig. 3(b)] we can estimate a FWHM of the LO-phonon resonance of $0.7(0.1) \mathrm{meV}$, which is of particular interest for a possible realization of the quantum interference involving remote QD sources [20,43]. The LO-phonon energy itself is hereby found in a range of $\hbar \omega_{\mathrm{LO}}=36.5 \pm 0.3 \mathrm{meV}$ in our QDs.

Single-photon purity under LO-phonon-assisted excitation. More importantly, in Fig. 3(c), we report on the HanburyBrown-Twiss (HBT) measurement on the $\mathrm{X}$ photons under the LO-phonon excitation and extract a multiphoton probability of $g_{X_{\mathrm{LO}}}^{(2)}(0)=1.9(0.1) \times 10^{-2}$, which is lower or equal than the multiphoton probabilities observed for the other excitation schemes explored here ([see Fig. 3(d)] but without any polarization suppression. This value might be still deteriorated by the contribution of the long-decay channel as observed in the second-order correlation measurement performed under $p$-shell excitation. Thus one could expect the multiphoton emission probability to be further lowered once more ideal conditions for the exciton-phonon coupling are elaborated or by introducing moderate time gating during the measurement process. Another intriguing effect is the correlation statistics under the LO-phonon excitation on timescales comparable to the laser pulse frequency. At first glance, one might assume strong QD blinking to be present $[44,45]$, however, if we compare the second-order correlation statistics on long timescales to a truly blinking emitter possibly induced under $p$-shell excitation (see Fig. S1 in Ref. [28]), we can conclude (a)

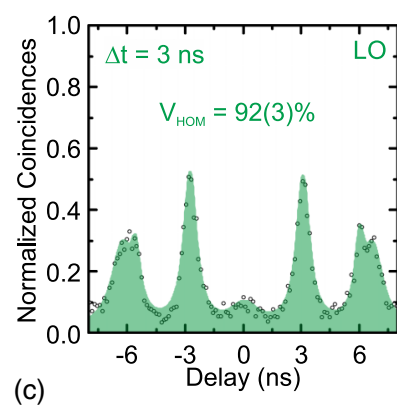

(b)

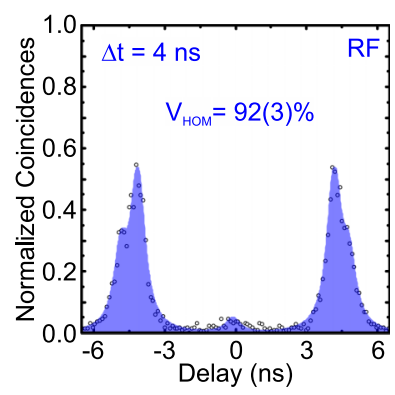

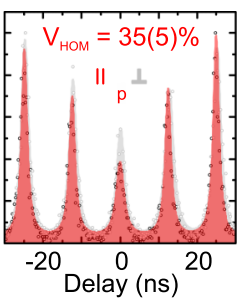

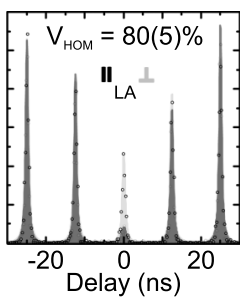

FIG. 4. (a) Two-photon interference measurement on $\mathrm{X}$ photons using co-polarized settings under LO-phonon excitation. The time gap $\Delta t$ between subsequently generated single photons used to probe the interference of the same source is $3 \mathrm{~ns}$. The quoted value of $V_{\mathrm{HOM}}$ is obtained by fitting the resulting histograms with Lorentzian peaks (resulting in a necessary background substraction for the given $\Delta t$ ) and by taking into account the nonideal properties of the interference beam-splitter. (b) Similar measurement but under RF excitation and slighlty increased $\Delta t$ of $4 \mathrm{~ns}$ between subsequently excited single photons. (c) Comparative study under LO-phonon (green), $p$-shell (red), and LA-phonon (black) excitations with $\Delta t=12 \mathrm{~ns}$. All data points are again fitted assuming Lorentzian peaks to obtain the depicted values of the interference visibility. The cross-polarized setting (grey) is additionally depicted in each panel.

that this correlation effect origins from a different mechanism. The fact that, at least on a very short timescale, the LO-phonon interaction provides an effective screening against blinking effects is highly interesting but requires additional experimental work to be performed as a function of a controlled charge environment by, for example, embedding the QDs in diode structures.

Indistinguishability among photons subsequently emitted by a GaAs $Q D$. The close to zero multiphoton emission probability combined to the fast intrinsic transition time present in weakly confined QDs should be beneficial to achieve highly indistinguishable photons even without embedding the QDs into sophisticated photonic structures $[1,2,36]$. To this end, we excite the QD with laser pulses separated by $\Delta t=3 \mathrm{~ns}$ and compensate this delay probabilistically with an unbalanced Mach-Zender interferometer. The result of a Hong-OuMandel (HOM) measurement for the LO-phonon excitation is shown in Fig. 4(a) and reveals an interference visibility of $V_{X_{\mathrm{LO}}}^{\mathrm{HOM}}=92(3) \%$ compared to the case of completely distinguishable photons (next neighboring peaks or cross-polarized measurements presented in Fig. S2 in Ref. [28] for completeness). Additionally, the indistinguishability under RF excitation is reported in Fig. 4(b) to gauge the effect of external reservoir pumping, where a comparable visibility of $V_{X_{\mathrm{RF}}}^{\mathrm{HOM}}=$ $92(3) \%$ (at larger excitation pulse separation of $\Delta t=4 \mathrm{~ns}$ ) 
is observed. We want to emphasize that the evaluation of the visibility relies on a phenomenological fit of the histograms data by applying Lorentzian fits to the correlation peaks in both cases, as in Refs. [12,20]. A more physical approach would include a sophisticated model for the long-decay channel in case of LO-phonon excitation and a proper evaluation of the FSS-induced state evolution present under RF. In view of the relatively large statistical uncertainties in the measurements, however, theoretical modeling (see Ref. [43]) returns visibility values comparable with Lorentzian fitting as summarized in Note 3 in Ref. [28] for the interested reader. The indistinguishability of X photons from several other QDs under LO-phonon excitation is also reported (see Fig. S3 in Ref. [28]) to prove the general validity of our result. It shows that the dressing of LO phonons induces a negligible amount of excitation time jitter, since it can readily compete with values observed under strict resonant condition and is on par to the best results reported for conventional InGaAs QDs under resonance fluorescence [22]. This result is important bearing in mind that the value is achieved without the Purcell enhancement or phonon sideband filtering [46]. Cavity structures featuring the Purcell enhancement and phonon sideband filtering could therefore enable near-unity indistinguishable photon emission even for the LO-phonon excitation scheme. Furthermore, the indistinguishability values are significantly higher than those reported for InGaAs QDs under LO-phonon excitation $(\approx 70 \%$ [27]), which we tentatively attribute to the high intrinsic transition rate of our weakly confining QDs-alleviating dephasing effects originated from phonon interaction and/or charge/spin noise [47]—and, eventually, the weaker overlap of the QD's confined states with the LOphonon resonance.

In a last step, we want to compare the LO-phonon excitation method to the other possible incoherent population schemes in our GaAs QDs. The time gap between subsequently excited single photons within the HOM interferometer is thereby enhanced to $12 \mathrm{~ns}$, which allows us to properly compare the visibility also in the case of a slow decay, i.e., observed under $p$-shell excitation. First, we repeat the measurement on the LO phonon [see Fig. 4(c)] and extract a visibility of $V_{X_{\perp \mathrm{O}}}^{\mathrm{HOM}}=78(4) \%$. We do observe a degradation of the indistinguishability, which we attribute to charge noise, as known from the literature to be often present already on these timescales $[12,48]$. It is particularly present in structures which do not alleviate the large bandwidth noise attributed to excess charge carriers in the QD environment via the Purcell enhancement [2]. A different picture is revealed in Fig. 4(c) under $p$-shell excitation. While under certain detuning conditions the spectra are dominated by the $\mathrm{X}$ transition and strongly resemble those obtained under LO-phonon excitation, the very long relaxation time and induced time jitter leads to photon indistinguishability values barely exceeding $30 \%$. This pronounced degradation has not been observed in InGaAs systems, where even under $p$-shell excitation the emission of indistinguishable photons $\left(V_{\mathrm{HOM}} \approx 90 \%\right.$ [49]) is realizable because of a slower radiative recombination rate combined with faster relaxation of the excited states. This allows us to conclude that, different from QDs featuring strong confinement, " $p$-shell" excitation is not a viable route to obtain photons with meaningful indistinguishability from weakly confining QDs. Instead, we focus once more on the resonant LA-phonon excitation. In Fig. 4(c), we report on a visibility of $V_{X_{L A}}^{\mathrm{HOM}}=80(5) \%$, compatible to the value obtained under LO-phonon excitation, which demonstrates that it is practically irrelevant which phonon decay channels are involved in droplet-etched GaAs QDs to obtain triggered indistinguishable single photons and once more underlines the advantage of the LO-phonon-assisted excitation.

\section{CONCLUSIONS AND PERSPECTIVES}

In this work, we have studied in detail possible optical excitation schemes to obtain highly indistinguishable single photons from neutral excitons confined in GaAs QDs obtained by the local droplet etching method. We summarize below the main results and discuss possible avenues opened by this work.

First, we have provided compelling evidence that excitons are weakly confined in such QDs based on the following observations: (i) the measured exciton lifetimes $(\approx 200$ $250 \mathrm{ps})$ are substantially shorter than the minimum expected for excitons strongly confined in GaAs QDs, (ii) nonradiative contributions to the measured decay time can be neglected because under excited-state-excitation decay times of up to $\approx 2 \mathrm{~ns}$ are observed, and (iii) the free-exciton Bohr radius is substantially smaller than the lateral extent of the confinement potential provided by GaAs-filled nanoholes.

The weak confinement has at least two important consequences: (i) an enhanced oscillator strength and (ii) a dense ladder of excited states, with typical energetic separation of a few meV. The intrinsically short lifetimes are beneficial to achieve high photon indistinguishability provided that the used laser pulses and the relaxation from the optically addressed state are substantially shorter. We find that the latter condition is not met for the generation of a ground-state exciton via excited states because of relaxation times, which are much longer than those typically reported for QDs in the strong confinement regime.

At present, we can only speculate on the origin of this anomalously long decay, which persists for all the addressed excited states and for above-band-gap excitation. Two plausible relaxation "bottlenecks" are proposed: (i) the high in-plane symmetry of our QDs might impose stringent requirements on the orbital/spin properties of the excited states for efficient relaxation and/or (ii) the fact that the energy separation between excited states is smaller than the LO-phonon energy might impose a phonon bottleneck. Further experiments accompanied by microscopic models capturing the dynamics of intradot exciton relaxation may shed light on this interesting phenomenon.

In contrast to the results obtained under $p$-shell and hstate excitation, highly indistinguishable photons are obtained under coherent RF and incoherent LA-phonon-assisted excitation, reaching a performance, which is at least on par with that of conventional InGaAs in the strong confinement regime. A technical problem with these excitation strategies is represented by the laser stray light rejection, which requires demanding polarization/spectral filtering, usually coming at the expense of source brightness. This motivated us to explore in detail the LO-phonon-assisted excitation, which has rarely 
been used for InGaAs QDs and only with modest results in terms of photon indistinguishability. When applied to our GaAs QDs, the LO-phonon-assisted excitation leads instead to HOM-interference visibilities comparable to those obtained under RF and LA-phonon-assisted excitation, with the noteworthy advantage of straightforward laser rejection. The efficient imprinting of the laser polarization on the polarization of the emitted photons, the relatively broad spectral width of the LO-phonon resonance, and the incoherent nature of the process are additional appealing features of this excitation scheme.

Our investigation leave however several fundamental and application-driven questions open: (i) TCSPC, HOM, HBT, polarization-resolved, and power-dependent measurements indicate the presence of a slow decay path, which competes with the desired fast decay of a LO-phonon-polaron into exciton plus LO phonon. Plausible explanations are that the photogeneration of a polaron competes with the creation of a spectrally overlapped excited exciton or that the polaron dissociates into an excited exciton and acoustic phonons. Further experimental and theoretical efforts are required to understand and possibly suppress this/these slow decay path/s. Experiments may involve both the engineering of the QD structural and optical properties $[34,50,51]$ and of the laserpulse properties. (ii) Because of the limited number of former experimental reports and the lack of microscopic models relating the LO-phonon-exciton coupling in QDs with different sizes/morphologies we cannot draw conclusions on whether the weak confinement is pivotal to the effectiveness of this excitation scheme and whether similar performance can be achieved on other QD systems. (iii) Our HBT measurements under pulsed excitation reveal not only competitive single-photon purity values but also an intriguing bunching at timescales corresponding to the pulse delay of $\approx 12 \mathrm{~ns}$. Here we dare to speculate that the LO phonon left behind by the polaron decay remains in the QD and enhances the absorption of the next pulse because of the pronounced 2LO-phonon resonance [52]. This is in principle possible because of the negligible group velocities of optically generated LO-phonons but would imply an extremely long LO-phonon lifetime.

Finally we would like to stress that in our study, we have focused on QDs embedded in simple planar cavities with modest light throughput to explore the intrinsic performance of GaAs QDs as emitters of indistinguishable photons. The integration of such QDs into advanced photonic structuresin particular highly efficient broadband cavities adequate for LO-phonon excitation [21] — can possibly ensure indistinguishable photons on arbitrary timescales and position GaAs QDs, or general weakly confined QDs, as excellent sources of upcoming quantum photonic networks offering practicable excitation schemes

\section{ACKNOWLEDGMENTS}

We acknowledge fruitful discussions with Y. H. Huo, M. Munsch, R. Warburton, G. Weihs, K. D. Jöns, and P. Klenovský. We further acknowledge H. Huang for help with the sample growth. This work was financially supported by the Austrian Science Fund (FWF): P 29603, the Linz Institute of Technology, the European Research Council (ERC) under the European Unions Horizon 2020 research and innovation programme (SPQRel, Grant Agreement No. 679183) and European Union Seventh Framework Programme (FP7/20072013) under Grant Agreement No. 601126 (HANAS). The authors would like to thank the DFG for financial support via the project Mi500/27-1. The research of the $\mathrm{IQ}^{S T}$ was financially supported by the Ministry of Science, Research and Arts Baden-Württemberg.
[1] N. Somaschi, V. Giesz, L. De Santis, J. C. Loredo, M. P. Almeida, G. Hornecker, A. Lemaítre, A. Auffeves, A. G. White, L. Lanco, and P. Senellart, Nat. Photonics 10, 340 (2016).

[2] X. Ding, Y. He, Z.-C. Duan, N. Gregersen, M.-C. Chen, S. Unsleber, S. Maier, C. Schneider, M. Kamp, S. Höfling, C.-Y. Lu, and J.-W. Pan, Phys. Rev. Lett. 116, 020401 (2016).

[3] M. E. Reimer, G. Bulgarini, N. Akopian, M. Hocevar, M. B. Bavnick, M. A. Verheijen, E. P. A. M. Bakkers, L. P. Kouwenhoven, and V. Zwiller, Nat. Commun. 3, 737 (2012).

[4] L. C. Andreani, G. Panzarini, and J.-M. Gérard, Phys. Rev. B 60, 13276 (1999).

[5] J. P. Reithmaier, Semicond. Sci. Technol. 23, 123001 (2008).

[6] E. Peter, P. Senellart, D. Martrou, A. Lemaître, J. Hours, J. M. Gérard, and J. Bloch, Phys. Rev. Lett. 95, 067401 (2005).

[7] A. Rastelli, S. Stufler, A. Schliwa, R. Songmuang, C. Manzano, G. Costantini, K. Kern, A. Zrenner, D. Bimberg, and O. G. Schmidt, Phys. Rev. Lett. 92, 166104 (2004).

[8] C. Heyn, A. Stemmann, T. Köppen, C. Strelow, T. Kipp, M. Grave, S. Mendach, and W. Hansen, Appl. Phys. Lett. 94, 183113 (2009).

[9] Y. H. Huo, A. Rastelli, and O. G. Schmidt, Appl. Phys. Lett. 102, 152105 (2013).
[10] S. Stobbe, T. W. Schlereth, S. Höfling, A. Forchel, J. M. Hvam, and P. Lodahl, Phys. Rev. B 82, 233302 (2010).

[11] D. Huber, M. Reindl, S. F. Covre da Silva, C. Schimpf, J. Martín-Sánchez, H. Huang, G. Piredda, J. Edlinger, A. Rastelli, and R. Trotta, Phys. Rev. Lett. 121, 033902 (2018).

[12] D. Huber, M. Reindl, Y. Huo, H. Huang, J. S. Wildmann, O. G. Schmidt, A. Rastelli, and R. Trotta, Nat. Commun. 8, 15506 (2017).

[13] R. Keil, M. Zopf, Y. Chen, B. Höfer, J. Zhang, F. Ding, and O. G. Schmidt, Nat. Commun. 8, 15501 (2017).

[14] L. Schweickert, K. D. Jöns, K. D. Zeuner, S. F. Covre da Silva, H. Huang, T. Lettner, M. Reindl, J. Zichi, R. Trotta, A. Rastelli, and V. Zwiller, Appl. Phys. Lett. 112, 093106 (2018).

[15] M. Reindl, D. Huber, C. Schimpf, S. F. Covre da Silva, M. Rota, H. Huang, V. Zwiller, K. Jöns, A. Rastelli, and R. Trotta, Sci. Adv. 4, eaau1255 (2018).

[16] F. Basso Basset, M. B. Rota, C. Schimpf, D. Tedeschi, K. D. Zeuner, S. F. Covre da Silva, M. Reindl, V. Zwiller, K. D. Jöns, A. Rastelli, and R. Trotta, arXiv:1901.06646 [Phys. Rev. Lett. (to be published)].

[17] H. de Riedmatten, I. Marcikic, W. Tittel, H. Zbinden, D. Collins, and N. Gisin, Phys. Rev. Lett. 92, 047904 (2004). 
[18] J. C. Loredo, N. A. Zakaria, N. Somaschi, C. Anton, L. de Santis, V. Giesz, T. Grange, M. A. Broome, O. Gazzano, G. Coppola, I. Sagnes, A. Lemaitre, A. Auffeves, P. Senellart, M. P. Almeida, and A. G. White, Optica 3, 433 (2016).

[19] H. Vural, S. L. Portalupi, J. Maisch, S. Kern, J. H. Weber, M. Jetter, J. Wrachtrup, R. Löw, I. Gerhardt, and P. Michler, Optica 5, 367 (2018).

[20] M. Reindl, K. D. Jöns, D. Huber, C. Schimpf, Y. Huo, V. Zwiller, A. Rastelli, and R. Trotta, Nano Lett. 17, 4090 (2017).

[21] J. Liu, R. Su, Y. Wei, B. Yao, S. F. Covre da Silva, Y. Yu, J. Iles-Smith, K. Srinivasan, A. Rastelli, J. Li, and X. Wang, Nat. Nanotechnol. 14, 586 (2019).

[22] Y.-M. He, Y. He, Y.-J. Wei, A. M. Wu Dian, C. Schneider, S. Höfling, M. Kamp, C.-Y. Lu, and J.-W. Pan, Nat. Nanotechnol. 8, 213 (2013)

[23] S. Gerhardt, M. Deppisch, S. Betzold, T. H. Harder, T. C. H. Liew, A. Predojevic, S. Höfling, and C. Schneider, Phys. Rev. B 100, 115305 (2019).

[24] Y.-M. He, H. Wang, S. Gerhardt, K. Winkler, J. Jurkat, Y. Yu, M.-C. Chen, X. Ding, S. Chen, J. Quian, Z.-C. Duan, J.-P. Li, L.-J. Wang, Y.-H. Huo, S. Yu, S. Höfling, C.-Y. Lu, and J.-W. Pan, arXiv:1809.10992.

[25] P.-L. Ardelt, L. Hanschke, K. A. Fischer, K. Müller, A. Kleinkauf, M. Koller, A. Bechtold, T. Simmet, J. Wierzbowski, H. Riedl, G. Abstreiter, and J. J. Finley, Phys. Rev. B 90, 241404(R) (2014).

[26] J. H. Quilter, A. J. Brash, F. Liu, M. Glässl, A. M. Barth, V. M. Axt, A. J. Ramsay, M. S. Skolnick, and A. M. Fox, Phys. Rev. Lett. 114, 137401 (2015).

[27] M. A. Pooley, D. J. P. Ellis, R. B. Patel, A. J. Bennett, K. H. A. Chan, I. Farrer, D. A. Ritchie, and A. J. Shields, Appl. Phys. Lett. 100, 211103 (2012).

[28] See Supplemental Material at http://link.aps.org/supplemental/ 10.1103/PhysRevB.100.155420 for the used methods, supplementary figures and fitting procedures of the Hong-Ou-Mandel interference.

[29] L. N. Tripathi, Y.-M. He, L. Dusanowski, P. A. Wroński, C.-Y. Lu, C. Schneider, and S. Höfling, Appl. Phys. Lett. 113, 021102 (2018).

[30] A. C. Dada, T. S. Santana, R. N. E. Malein, A. Koutroumanis, Y. Ma, J. M. Zajac, J. Y. Lim, J. D. Song, and B. D. Gerardot, Optica 3, 493 (2016).

[31] E. Schöll, L. Hanschke, L. Schweickert, K. D. Zeuner, M. Reindl, S. F. Cover da Silva, T. Lettner, R. Trotta, J. J. Finley, K. Müller, A. Rastelli, V. Zwiller, and K. D. Jöns, Nano Letters 19, 2404 (2019).

[32] F. Liu, L. M. P. Martins, A. J. Brash, A. M. Barth, J. H. Quilter, V. M. Axt, M. S. Skolnick, and A. M. Fox, Phys. Rev. B 93, 161407(R) (2016).

[33] J.-P. Jahn, M. Munsch, L. Béguin, A. V. Kuhlmann, M. Renggli, Y. Huo, F. Ding, R. Trotta, M. Reindl, O. G. Schmidt, A. Rastelli, P. Treutlein, and R. J. Warburton, Phys. Rev. B 92, 245439 (2015).
[34] H. Huang, R. Trotta, Y. Huo, T. Lettner, J. S. Wildmann, J. Martín-Sánchez, D. Huber, M. Reindl, J. Zhang, E. Zallo, O. G. Schmidt, and A. Rastelli, ACS Photonics 4, 868 (2017).

[35] E. Stock, M.-R. Dachner, T. Warming, A. Schliwa, A. Lochmann, A. Hoffmann, A. I. Toropov, A. K. Bakarov, I. A. Derebezov, M. Richter, V. A. Haisler, A. Knorr, and D. Bimberg, Phys. Rev. B 83, 041304(R) (2011).

[36] F. Liu, A. J. Brash, J. O’Hara, L. M. P. P. Martins, C. L. Phillips, R. J. Coles, B. Royall, E. Clarke, C. Bentham, N. Prtljaga, I. E. Itskevich, L. R. Wilson, M. S. Skolnick, and A. Mark Fox, Nat. Nanotechnol. 13, 835 (2018).

[37] J. Seebeck, T. R. Nielsen, P. Gartner, and F. Jahnke, Phys. Rev. B 71, 125327 (2005)

[38] P. Gartner, J. Seebeck, and F. Jahnke, Phys. Rev. B 73, 115307 (2006).

[39] T. Grange, R. Ferreira, and G. Bastard, Phys. Rev. B 76, 241304(R) (2007).

[40] Y. Toda, O. Moriwaki, M. Nishioka, and Y. Arakawa, Phys. Rev. Lett. 82, 4114 (1999).

[41] A. Reigue, A. Lemaître, C. Gomez Carbonell, C. Ulysse, K. Merghem, S. Guilet, R. Hostein, and V. Voliotis, Appl. Phys. Lett. 112, 073103 (2018).

[42] M. Cosacchi, F. Ungar, M. Cygorek, A. Vagov, and V. M. Axt, Phys. Rev. Lett. 123, 017403 (2019).

[43] J. H. Weber, B. Kambs, J. Kettler, S. Kern, J. Maisch, H. Vural, M. Jetter, S. L. Portalupi, C. Becher, and P. Michler, Nat. Nanotechnol. 14, 23 (2019).

[44] K. D. Jöns, K. Stensson, M. Reindl, M. Swillo, Y. Huo, V. Zwiller, A. Rastelli, R. Trotta, and G. Björk, Phys. Rev. B 96, 075430 (2017).

[45] J. H. Weber, J. Kettler, H. Vural, M. Müller, J. Maisch, M. Jetter, S. L. Portalupi, and P. Michler, Phys. Rev. B 97, 195414 (2018).

[46] J. Iles-smith, D. P. S. Mccutcheon, A. Nazir, and J. Mørk, Nat. Photonics 11, 521 (2017).

[47] A. Kiraz, M. Atatüre, and A. Imamoğlu, Phys. Rev. A 69, 032305 (2004).

[48] A. Thoma, P. Schnauber, M. Gschrey, M. Seifried, J. Wolters, J.-H. Schulze, A. Strittmatter, S. Rodt, A. Carmele, A. Knorr, T. Heindel, and S. Reitzenstein, Phys. Rev. Lett. 116, 033601 (2016).

[49] G. Kiršanskè, H. Thyrrestrup, R. S. Daveau, C. L. Dreeßen, T. Pregnolato, L. Midolo, P. Tighineanu, A. Javadi, S. Stobbe, R. Schott, A. Ludwig, A. D. Wieck, S. I. Park, J. D. Song, A. V. Kuhlmann, I. Söllner, M. C. Löbl, R. J. Warburton, and P. Lodahl, Phys. Rev. B 96, 165306 (2017).

[50] S. Kumar, R. Trotta, E. Zallo, J. D. Plumhof, P. Atkinson, A. Rastelli, and O. G. Schmidt, Appl. Phys. Lett. 99, 161118 (2011).

[51] X. Yuan, F. Weyhausen-Brinkmann, J. Martín-Sánchez, G. Piredda, V. Křápek, Y. Huo, H. Huang, C. Schimpf, O. G. Schmidt, J. Edlinger, G. Bester, R. Trotta, and A. Rastelli, Nat. Commun. 9, 3058 (2018).

[52] F. Findeis, A. Zrenner, G. Böhm, and G. Abstreiter, Phys. Rev. B 61, R10579(R) (2000). 